\title{
Sequential Chemoradiation
}

National Cancer Institute

\section{Source}

National Cancer Institute. Sequential Chemoradiation. NCI Thesaurus. Code C141341.

Treatment in which radiation therapy is administered after the completion of chemotherapy. 